\title{
The Role of Oxygen at Screw Dislocations in GaN
}

\author{
I. Arslan ${ }^{1}$ and N. D. Browning 2,3 \\ ${ }^{1}$ Department of Physics, University of California-Davis, 1 Shields Ave. Davis, CA 95616 \\ ${ }^{2}$ Department of Chemical Engineering and Materials Science, University of California-Davis, \\ Davis, CA 95616 \\ ${ }^{3}$ National Center for Electron Microscopy, Lawrence Berkeley National Laboratory, \\ Berkeley, CA 94720
}

\begin{abstract}
Here we report the first direct atomic scale experimental observations of oxygen segregation to screw dislocations in $\mathrm{GaN}$ using correlated techniques in the scanning transmission electron microscope. The amount of oxygen present in each of the three distinct types of screw dislocation core is found to depend on the evolution and structure of the core, and thus gives rise to a varying concentration of localized states in the band-gap. Contrary to previous theoretical predictions, the substitution of oxygen for nitrogen is observed to extend over many monolayers for the open core dislocation.
\end{abstract}


In recent years, $\mathrm{GaN}$ has developed into an important semiconductor for optical and electronic applications. However, due to a large lattice mismatch with the substrate, the material possesses an overall high density of threading dislocations. While efforts have been made to reduce dislocation density, the remaining dislocations still play an important role in device performance, one that is still not well understood. The previous work of Nakamura and others in fabricating GaN-based blue and green light emitting diodes (LED's) and commercializing nitride laser diodes [1,2] has proven functionality for $\mathrm{GaN}$ devices irrespective of impurities and dislocations, but an explanation of changes in device performance and efficiency over time is still pending. In fact, most purely intrinsic dislocations appear not to be related to device failure $[3,4]$. It therefore appears that the interaction of dislocations with impurities, and in particular, oxygen, may create impurity states in the band gap, act as traps for carriers, and could prove to be one of the key sources of device breakdown.

Oxygen is an impurity that is known to be present in GaN thin films. However, as there exist only a few experimental studies of oxygen in $\mathrm{GaN}$, the fundamental origin and role of the oxygen in determining the overall electronic characteristics of the films is poorly understood. For example, it has been suggested that oxygen in $\mathrm{GaN}$ may originate as an impurity in the $\mathrm{NH}_{3}$ precursor for metal organic chemical vapor deposition (MOCVD), or may originate from the remnant water vapor in molecular beam epitaxy (MBE) [5]. Another suggestion is that oxygen from the $\mathrm{Al}_{2} \mathrm{O}_{3}$ substrate may diffuse within the disordered interfacial region, creating donors responsible for the formation of 
an impurity band [6]. Furthermore, Chung and Gershenzon showed that the amount of oxygen in the growth process has a strong influence on the carrier concentration, acting as a shallow donor and creating an impurity band about $78 \mathrm{meV}$ below the conduction band [7]. Secondary ion-mass spectroscopy measurements show that GaN can contain oxygen at least in the range of $10^{16}-10^{17}$ per $\mathrm{cm}^{3}$ [8]. However, Mattila and Nieminen suggest that a significant portion of this implanted oxygen resides at interstitial sites, thus remaining electrically inactive [9].

A further complication to understanding the complex role of oxygen is how it interacts with the abundant dislocation cores in $\mathrm{GaN}$, and whether the combination of impurity plus dislocation produces either a deleterious or benign effect. In this letter, we address the issue of impurity segregation to one of the three types of dislocations in GaN, the screw dislocation. More specifically, we provide direct experimental observations on the segregation of oxygen to three variants of screw dislocations: open core, filled core, and full core, and its effect on the electronic structure. These measurements provide evidence in favor of the interaction of oxygen with screw dislocations providing the origin of localized states at the cores.

The experimental techniques used in this analysis are Z-contrast imaging and electron energy loss spectroscopy (EELS) performed in a JEOL 2010F 200kV scanning transmission electron microscope (STEM) [10]. In a Z-contrast image the scattered intensity that is detected at high angles and over a large angular range averages coherent effects between atomic columns in the specimen, thus allowing each atom to be considered to scatter independently with a cross section approaching a $Z^{2}$ dependence on atomic number (and hence the name Z-contrast imaging). This detection geometry thus 
yields an incoherent image where changes in focus and thickness do not cause contrast reversals in the image (unlike conventional phase-contrast high-resolution transmission electron microscopy (HREM)), so that atomic sites can be identified unambiguously during the experiment. Another advantage of the STEM is the ability to perform EELS simultaneously with the image acquisition. Inelastically scattered electrons will scatter to lower angles and be dispersed based on the energy they lose while traveling through the specimen. This energy lost by the fast electrons is the same amount of energy transferred to the electrons in the material, promoting them to higher unoccupied states. Therefore, the peaks in the energy-loss spectrum map the density of unoccupied states in the material. As the annular detector used for Z-contrast imaging does not interfere with the low-angle scattering used for EELS, this means that the Z-contrast image can be used to position the electron probe over a particular structural feature for acquisition of a spectrum. The GaN samples analyzed using these techniques are metal organic chemical vapor deposition (MOCVD) grown n-type (Si-doped) samples on a sapphire substrate. As mentioned earlier, the MOCVD growth method makes the samples vulnerable to oxygen impurities during growth, as well as oxygen that might diffuse from the sapphire substrate. The absolute level of unintentional oxygen impurities in these samples is uncharacterized. However, the segregation effects observed here will simply occur on a greater or lesser extent as the level of impurities changes. It is also important to note that no oxygen impurities were observed in the bulk.

The first type of core analyzed was the open core screw dislocation, also known as a nanopipe. The particular nanopipe we show here is somewhat large, being $\sim 45 \mathrm{~nm}$ in diameter (Fig. 1(a)). The higher magnification image (Fig. 1(b)) shows the atomic 
structure in the region of the core and also the positions where EEL spectra were acquired (Fig. 1(c)). Clearly, as the probe is moved in toward the edge of the nanopipe, the nitrogen signal in the EEL spectrum decreases while the oxygen signal increases. Finally, at the very edge of the nanopipe, only an oxygen signal remains, indicating the formation of $\mathrm{GaO}$ lining the walls of the nanopipe. This result provides strong evidence that oxygen substitutes for nitrogen on the lattice sites, and not only specifically at the edge, but even further in towards the bulk of the sample, $\sim 60 \AA$. If the oxygen was at interstitial positions, the nitrogen signal would remain constant and the Z-contrast image would change dramatically in contrast and resolution (interstitials would introduce strain and reduce the ability to image the lattice directly). These observations do not necessarily contradict the idea that there may be some critical saturation of oxygen that stunts nanopipe growth, nor does it contradict that the nanopipe walls may be a way for the oxygen to diffuse into the bulk material.

This experimental evidence supports the simulations of Jones et al. that suggest that the oxygen has a tendency to segregate to the (10-10) surface to form $\mathrm{V}_{\mathrm{Ga}}-\left(\mathrm{O}_{\mathrm{N}}\right)_{3}$ defects [11]. While a quantitative determination of the amount of Ga vacancies was not performed here, the decrease in Z-contrast image intensity towards the edge of the nanopipe and observation of oxygen on nitrogen sites is certainly in agreement with their theory. However, their simulations also indicate that further growth of the nanopipe is stunted when a critical concentration of oxygen on the order of a monolayer is reached. Our experimental observations show clear and direct evidence that the oxygen is not only present at one monolayer, but extends $\sim 20$ monolayers into the film (although decreasing 
in concentration further into the sample). This experimental result is reproducible, and yields the same result for different sized nanopipes in MOCVD samples.

The second type of core analyzed was a closed, or filled nanopipe, $\sim 9 \mathrm{~nm}$ in diameter (Fig. 2(a)). Although the hexagonal structure of $\mathrm{GaN}$ is visible everywhere in the image, there is a dark contrast in the middle of the nanopipe. This suggests that this dislocation was originally an open core nanopipe, and that material filled in the nanopipe at a later stage of growth. A similar observation has been made by Stach et al., where as GaN was cooled from elevated temperatures, the previously empty cores were filled with liquid $\mathrm{Ga}$ which then reacted with gaseous nitrogen to form $\mathrm{GaN}$ [12]. This result could also be consistent with the pinholes observed by Liliental-Weber et al. where the dislocation is observed to open and close during growth [13].

From the contrast in the image, it appears that the center does not have the same thickness as the surrounding bulk material, which can also be supported by the decrease in intensity in the EELS signal, as compared to the bulk [3]. Further, there is an even darker spot of contrast exactly in the center of the filled nanopipe. This is presumably the center of the screw dislocation, and therefore, the EELS analysis was done in three sections, as labeled in the image. Fig. 2(b) shows the corresponding nitrogen and oxygen K-edges. The spectrum from position 1 (the center of the core) does not show a quantifiable amount of oxygen, as can be seen from the noise oscillations in figure 2(c). The spectra from positions 2 and 3 show an oxygen signal. The difference in intensity is caused by spectrum 2 sampling a face of the pinhole and spectrum 3 sampling purely an edge. This result suggests that oxygen had segregated to the core when it was open, and a small amount remained after the core had filled in. The low concentration of oxygen at 
this type of screw dislocation implies that it should have a lesser effect on the electrical properties than the open core dislocation. However, it should be noted that this concentration may vary from core to core and from sample to sample when the background level of oxygen impurities changes.

More detailed information on the electronic properties of the filled core screw dislocation may be obtained by analyzing the fine structure of the nitrogen K-edges (Fig. 2(d)). At position 1, there is a peak that is split with respect to the bulk spectrum, which is a signature of the screw dislocation [3]. At position 2, the fine structure has changed to appear more like the single peak of the bulk, which is expected, as we are moving away from the center of the core. However, while we would expect position 3 to resemble the bulk even more, it appears that the fine structure at this position has the characteristics of both positions 1 and 2. Furthermore, we observe that there is more intensity in the prepeak before the edge onset. One rational explanation for this may be the existence of a substantial amount of oxygen at position 3 , which is presumably forming states in the band gap below the conduction band onset. Although we do not have the energy resolution here to make a quantifiable statement about where exactly the states are formed in the gap, it appears from the spectrum that if states are formed, they might be at such a small energy below the onset that the impurity band might be merged into the conduction band to form a small set of continuous states in the band gap [7].

The third type of dislocation analyzed was a normal threading, or full core screw dislocation. These dislocations have never opened up to become a nanopipe, and are therefore very difficult to observe in a Z-contrast image (Fig. 3(a)). However, the small 
change in contrast is more apparent through a Fourier transform of the image (Fig. 3(b)), and it was from the center of this contrast that spectra were acquired (Fig 3(c)). The inset in Fig. 3(c) shows that there is minimal oxygen segregation to this type of core, and while a small amount may be present, it is below our detection limits. Additionally, by looking at the N K-edge, we can see that although the fine structure is not bulk-like, it has not resolved the two clear peaks characteristic to the screw dislocation, as shown in the simulation [3]. This is probably due to the contribution of bulk effects in the spectra from either a small amount of drift (changes in fine structure are highly localized to $3 \AA$ ), or simply because plural scattering in this thickness of specimen does not allow us to resolve the fine-structure with the required degree of localization (not the case for the previous 2 cores, where the structures were easily observable in both the image and spectrum).

In conclusion, we have observed for the first time on the atomic scale, oxygen segregation effects at three different types of screw dislocations in MOCVD grown samples: an open core, or nanopipe, a filled core, and a full core threading screw dislocation. Oxygen was found to be present at the edges of the open and filled nanopipes, having the strongest concentration at the surface layers, but continuing for approximately 20 monolayers into the sample for the open core. The center of the filled nanopipe and the full core screw dislocation did not show a quantifiable amount of oxygen. The presence of a pre-edge in the nitrogen spectrum at positions where there is appreciable oxygen present appears to confirm the link between oxygen and the creation of states in the band gap, thus causing the unwanted, deleterious electrical activity at the cores. 
This work was supported by LDRD funding through Lawrence Berkeley National Laboratory. The authors would like to thank Christian Kisielowski for the sample and preparation, and Eric Stach for his valuable comments on the filling of dislocation cores in $\mathrm{GaN}$. 


\section{REFERENCES:}

[1] S. Nakamura, T. Mukai, and M. Senoh, Appl. Phys. Lett. 64, 1687 (1994).

[2] S. Nakamura, Proc. SPIE 3628, 158 (1999).

[3] I. Arslan and N. D. Browning, Phys. Rev. B 65, 075310 (2002).

[4] A.T. Blumenau et. al., J. of Phys-Cond. Matt. 12, 10223 (2000).

[5] S. J. Pearton et. al., Appl. Phys. Lett. 75, 2939 (1999).

[6] C. Mavroidis et. al., Appl. Phys. Lett. 79, 1121 (2001).

[7] B-C. Chung and M. Gershenzon, J. Appl. Phys. 72, 651 (1992).

[8] W. Götz et. al., Appl. Phys. Lett. 68, 3144 (1996).

[9] T. Mattila and R. M. Nieminen, Phys. Rev. B 54, 16676 (1996).

[10] E. M. James and N. D. Browning, Ultramicroscopy 78, 125 (1999).

[11] R. Jones et. al., Phys. Stat. Sol. (a) 171, 167 (1999).

[12] E.A. Stach, private communication.

[13] Z. Liliental-Weber et. al., Phys. Rev. Letts. 79, 2835 (1997). 


\section{FIGURE CAPTIONS:}

Figure 1:

(a) A nanopipe that is $\sim 45 \mathrm{~nm}$ in diameter in MOCVD grown GaN. This open core screw dislocation is empty in the middle (dark area).

(b) Higher magnification image of one portion of one side of the nanopipe. The image has been marked with 5 positions where the probe was placed for EELS analysis.

(c) The EELS spectra from the 5 positions show that as the probe is placed closer to the edge of the nanopipe, the nitrogen signal decreases while the oxygen signal increases, extending $\sim 20$ monolayers away from the edge.

Figure 2:

(a) Image of a filled nanopipe, or filled core that is $\sim 90 \AA$ in diameter. The positions for the EELS analysis are marked on the image.

(b) The spectra from position 1 do not show a quantifiable oxygen signal above the noise, but the signal from positions 2 and 3 do show an oxygen edge, indicating that oxygen is still bonded at the edges of the nanopipe.

(c) A closer look at the background subtracted and smoothed oxygen $\mathrm{K}$ edge shows the differences in oxygen concentration from the three areas. Position 1 has no oxygen, as can be seen from the fluctuation of signal about the zero line (dotted line). Position 2 
shows oxygen is present although the peak is not well defined, and finally position 3 has the highest concentration. The difference in intensity is caused by spectrum 2 sampling a face of the pinhole and spectrum 3 sampling purely an edge.

(d) The fine structure of the $\mathrm{N} \mathrm{K}$ edge shows that position 1 has the signature of a screw dislocation, while position 2 appears to be bulk-like. However, the fine structure at position 3 seems not to be bulk-like, and a pre-peak has appeared, both as a result of oxygen at this position. The pre-peak indicates that oxygen is responsible for the formation of localized states in the band gap.

\section{Figure 3:}

(a) Z-contrast image of a full core screw dislocation.

(b) Inverse Fourier transform of the Z-contrast image to see the strain at the center of the core (brighter region).

(c) EELS spectra were acquired from this central bright position and are shown plotted against a simulation. The inset shows that there is no oxygen present in this closed core. 


\section{FIGURES:}

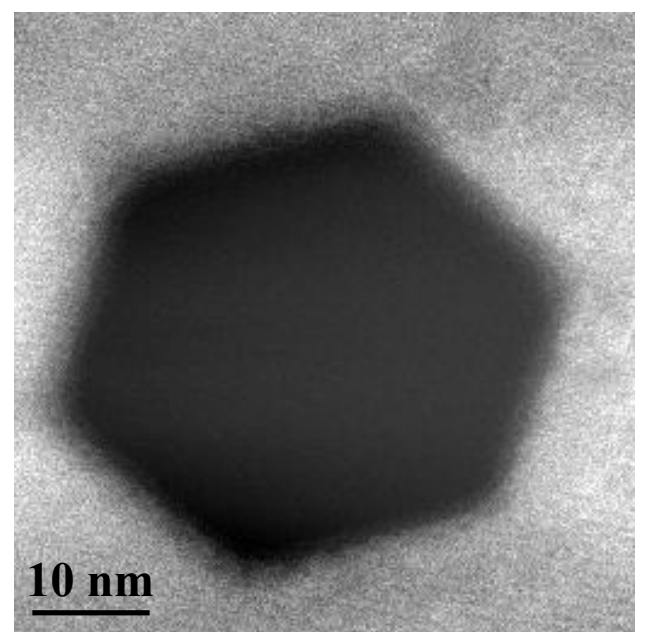

Figure 1(a).

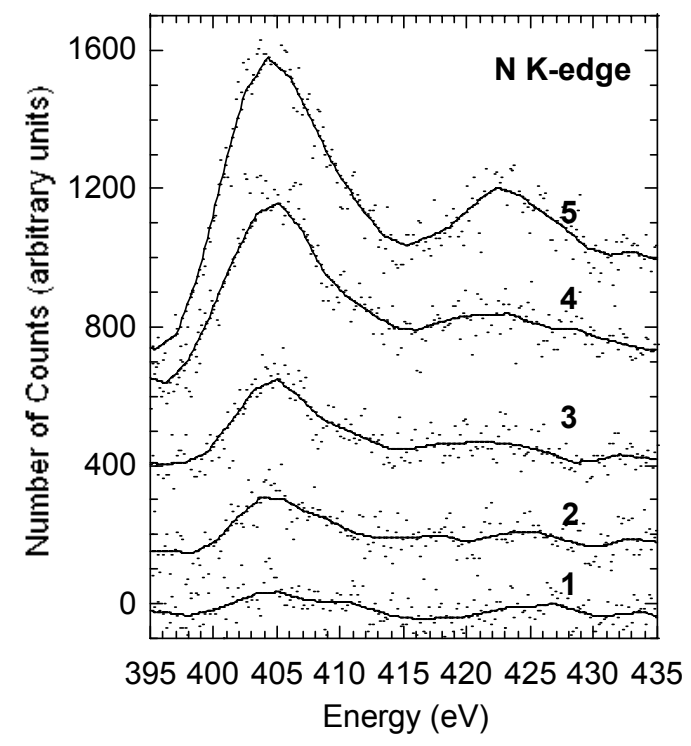

Figure 1(c).

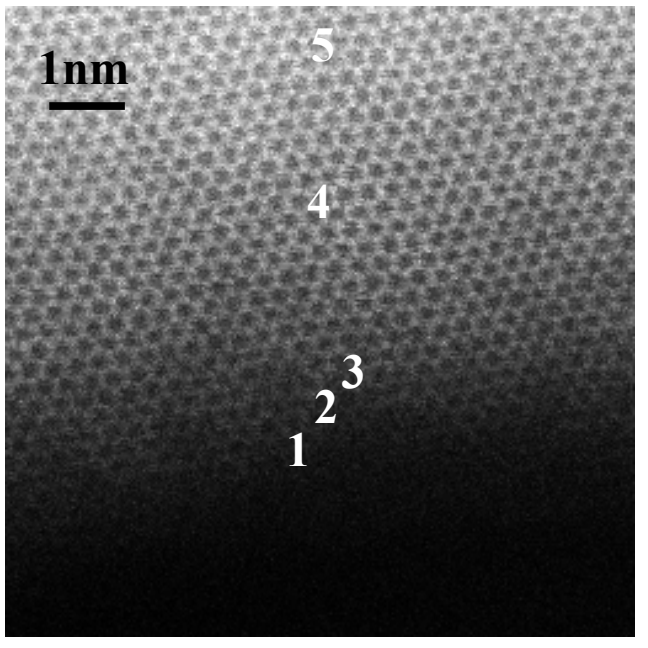

Figure 1(b).

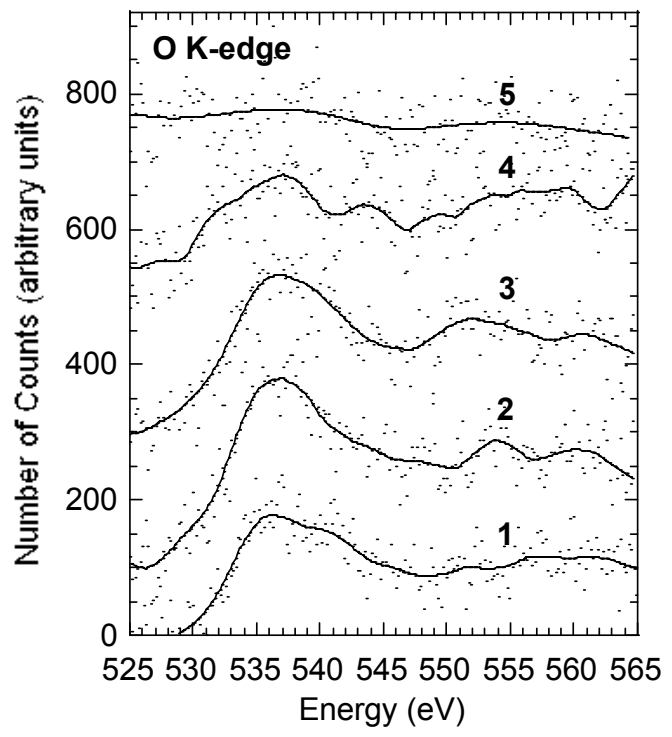




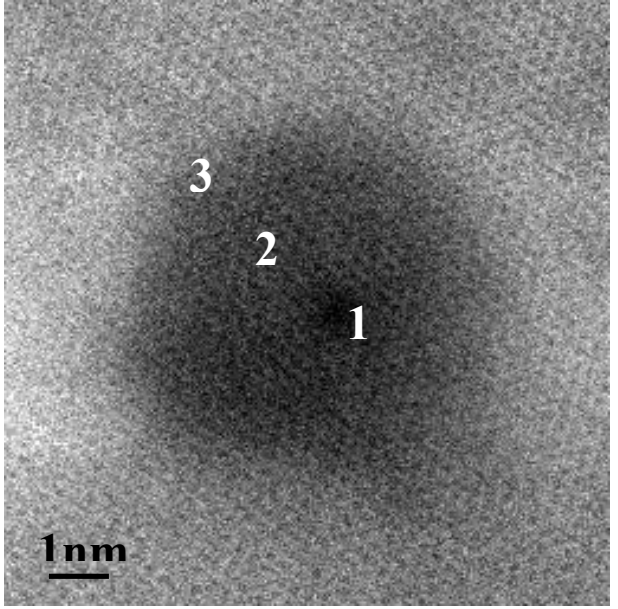

Figure 2(a).

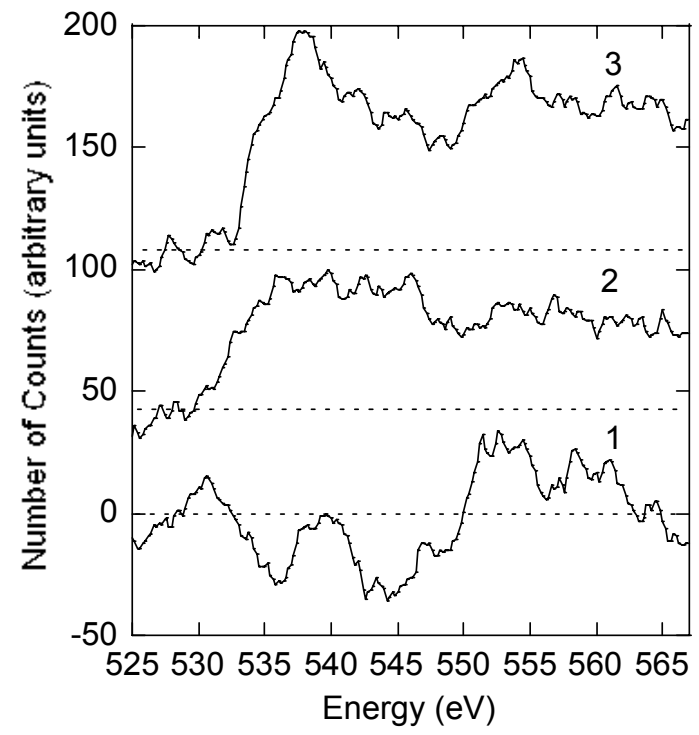

Figure 2(c).

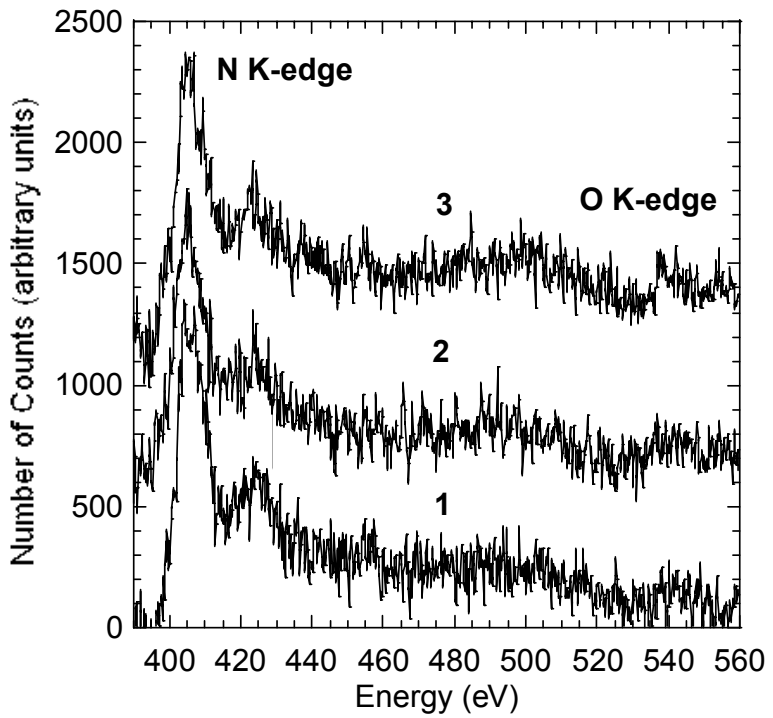

Figure 2(b).

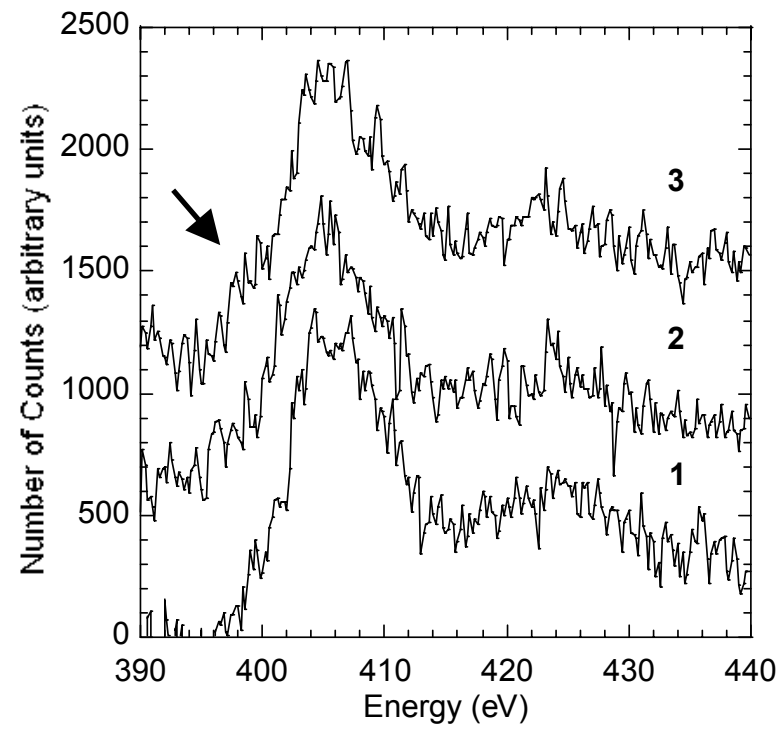

Figure 2(d). 
Figure 3(a).

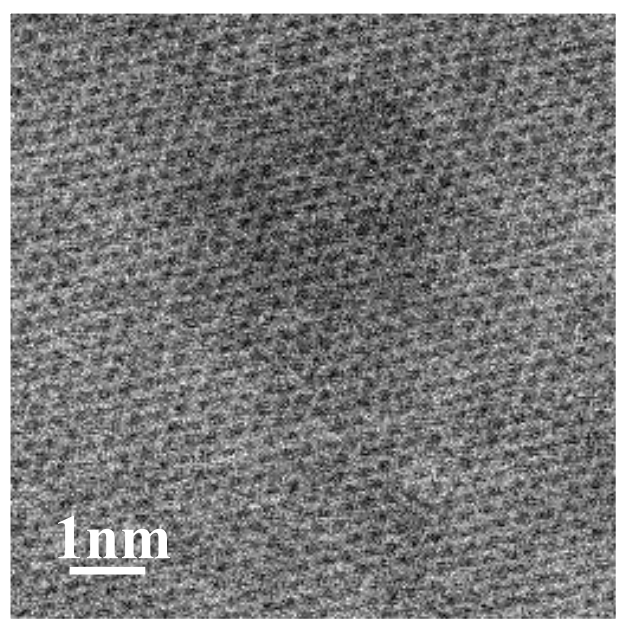

Figure 3(b).
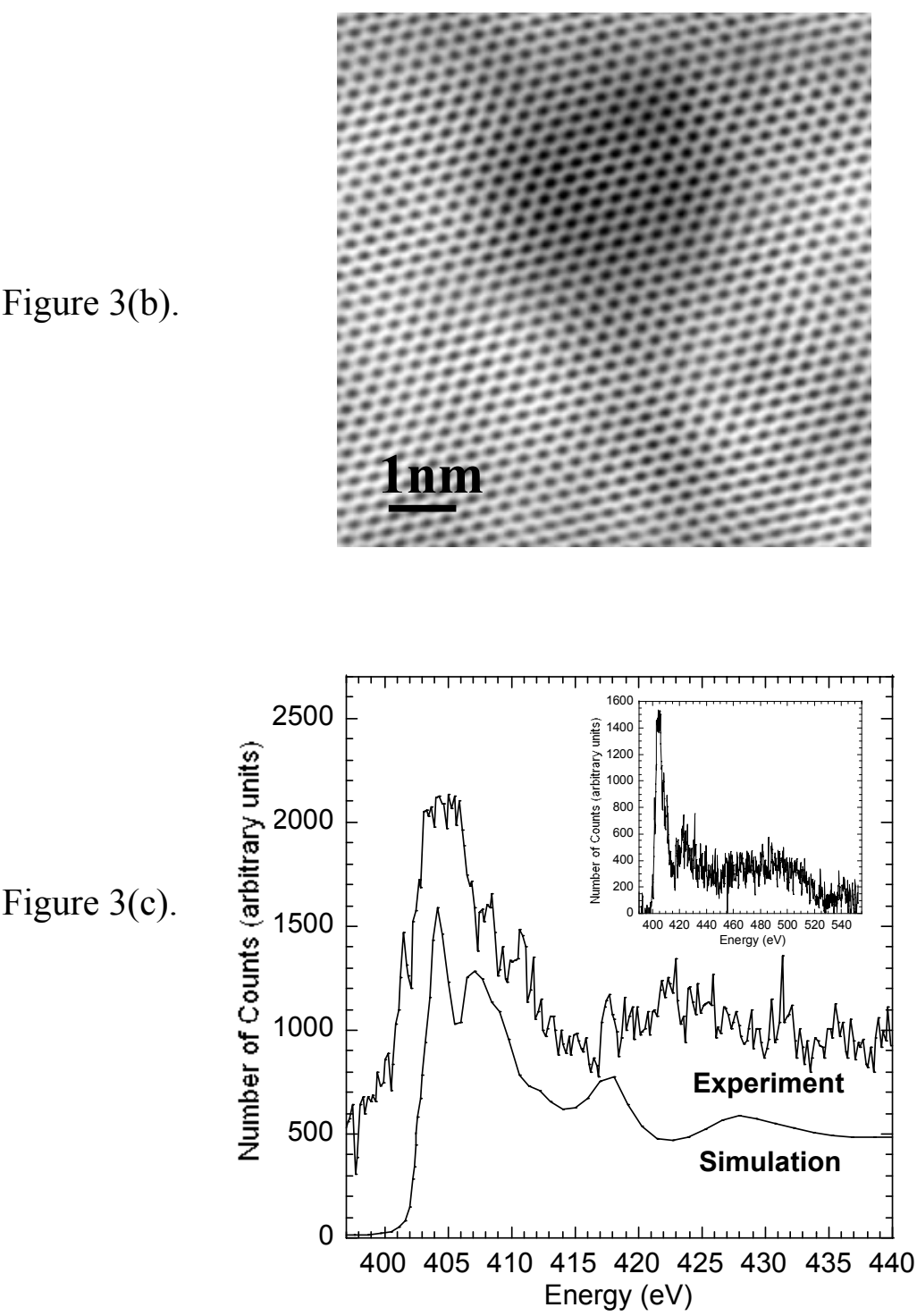